\title{
Computerized Cardiotocography: A Software to Generate Synthetic Signals
}

Improta G, Romano M, Ponsiglione A, Bifulco P, Faiella G and Cesarelli M*

DIETI, University of Naples "Federico II", Naples, Italy

\begin{abstract}
In recent years there has been an increasing interest in developing computerized methods to analyse cardiotocographic recordings, due to the widespread use of cardiotocography to record foetal heart rate and uterine activity signals and the recognized inter and intra-observer variability in the visual evaluation of these signals, in recent years there has been an increasing interest in developing computerized methods to analyse cardiotocographic recordings. As a result of this trend, new biomedical software and analysing algorithms have been enforced to replace the traditional interpretation of cardiotocographic traces. Artificial cardiotocographic signals play a predominant role in testing performance of these software. The present study presents a software for simulating synthetic cardiotocographic recordings developed in order to cover a wide range of physiological conditions and recording situations. It allows the simulation of the main features and characteristics of a real cardiotocographic trace (baseline fluctuation, accelerations, decelerations and contractions). Moreover, it permits the simulation of anomalies due to the acquisition system and/ or cardiac arrhythmia (signal loss and outliers). All the parameters used in the simulation can be set and modified at user choice. The proposed software, which generates synthetic signals resembling real cardiotocographic recordings, facilitates the comparison of different signal processing methods in order to establish their performances and could be employed also as a teaching tool for demonstration to medical students and others.
\end{abstract}

Keywords: Computerized Cardiotocography; Fetal monitoring; Fetal heart rate simulation

\section{Introduction}

At the end of pregnancy, assessing of foetal well-being depends on the evaluation of multiple parameters, many of them correlated to the characteristics of foetal heart rate (FHR) signals recorded by means of Cardiotocography (CTG), a widely used technique for antepartum and intrapartum foetal surveillance. This method refers to the continuous and simultaneous recording of FHR and Uterine Activity (UC signal) [1]. In this way, CTG provides continuous heart rate information; FHR changes in response to UC and other internal or external stimuli can be monitored and CTG is used as an indirect screening test for severe asphyxia (i.e., asphyxia severe enough to cause neurologic damage or foetal death) [1].

Even though CTG is extensively used in daily practice, studies of FHR reliability have shown significant intra-observer and interobserver variation in tracing interpretation $[2,3]$. As a result of the poor reproducibility of visual analysis of CTG traces and of the difficulties for obstetric staffs in evaluating foetal state [4,5], doubts arose regarding the efficacy of FHR monitoring in improving foetal outcome [6]. In order to decrease the subjective nature of foetal state evaluation and to develop objective means for CTG reading, the visual interpretation is often replaced by automated computerized analysis, which has been developed during the last two decades thanks to the technological advances in computers and in signal processing methods $[1,7,8]$.

Currently, new biomedical signal processing techniques and software are evaluated by processing real CTG signals $[9,10]$. However, because of the complexity and variability of real FHR signals, it is difficult to infer how the performance would vary in different clinical settings. Therefore, the availability of a large amount of realistic artificial CTG signals, with the possibility of easily changing their parameters and features, may facilitate the evaluation of software performances. Under this perspective, the development of software and models to generate synthetic signals with appropriate characteristics is a subject that has been widely investigated also for adult subjects $[11,12]$. Moreover, the usefulness of simulated heart rate traces with various characteristics for the assessment of diagnostic devices and analysing algorithms has been shown in different studies, both on adult and on foetal heart signals [13-15]. Encouraged from the above highlighted results, in this paper, we present a recent upgrade of a novel software for simulating CTG recordings relative to different foetal conditions and recording situations. The purpose of the proposed software is to provide a realistic CTG signal with reasonable waveform morphology features, which can be generated with specific statistics such as the mean and standard deviation of the FHR and frequency-domain characteristics of FHR variability (FHRV). In particular, we aimed to properly simulate the most relevant FHR features commonly evaluated in the clinical assessment of foetal health (i.e. accelerations, decelerations and contractions) along with the most troublesome anomalies due to either acquisition systems (e.g. signal loss) or physiological conditions (e.g. cardiac arrhythmias).

\section{Materials and Methods}

Synthetic CTG signals were artificially generated via software using Matlab R2011a. For FHR simulation, a slightly modified version of a method proposed for adults by other authors [16,17] and already employed in previous works concerning foetal monitoring was used [18-22]. Following this procedure, an artificial R-R tachogram with specific power spectrum characteristics, proper for foetal heart rhythm, was generated using an inverse Fourier transform [22]. After that, the

*Corresponding author: Cesarelli M, DIETI, University of Naples "Federico II", Naples, Italy, Tel: +39-081-768380; E-mail: cesarell@unina.it

Received May 27, 2014; Accepted August 05, 2014; Published August 25, 2014

Citation: Improta G, Romano M, Ponsiglione A, Bifulco P, Faiella G, et al. (2014) Computerized Cardiotocography: A Software to Generate Synthetic Signals. J Health Med Informat 5: 162. doi:10.4172/2157-7420.1000162

Copyright: $\odot 2014$ Improta G, et al. This is an open-access article distributed under the terms of the Creative Commons Attribution License, which permits unrestricted use, distribution, and reproduction in any medium, provided the original author and source are credited. 
FHR signal was computed using the known formula:

$$
F H R=\frac{k}{R R}
$$

where $k=60$ if $R R$ is expressed in seconds or $k=60000$ if $R R$ is expressed in ms. UC signals instead were generated directly in time domain.

It is worth underlining that all the parameters necessary for CTG simulation can be modified at user choice in order to cover a wide range of physiological conditions and recording situations.

\section{Power spectrum characteristics}

FHRV frequency characteristics are set by fixing the following parameters: boundaries of low frequency (LF) and high frequency (HF) bands; their standard deviation; LF/HF power ratio, a useful clinical parameter widely known as sympatho-vagal balance (SVB).

FHR settings: Mean FHR can be set within the range of normality (120-160 bpm); otherwise, other values exceeding this range can be chosen to simulate severe bradycardia or tachycardia, always getting realistic signals. Then, the simulation software allows to choose the standard deviation (SD) of FHR signal (in our tests the SD was set to obtain FHR of desired peak-peak amplitude). Besides, a SD varying along the FHR signal can be added, in order to simulate different alternating foetal states (sleep or awake, higher or lower activity).

\section{Baseline, Accelerations and Decelerations}

There is a common consensus that reassuring FHR patterns include each of the following characteristics: FHR baseline from 120 to $160 \mathrm{bpm}$, moderate variability and FHR accelerations appropriate for gestational age. Presence of FHR decelerations are generally suspicious findings [23]. In order to resemble real physiological situations, baseline fluctuation, accelerations and decelerations were simulated, adopting classical definitions [23]. In particular, we used a slowly varying sinusoid to simulate the FHR baseline that can be modified in amplitude and frequency, and, with regards to accelerations and decelerations, we used Gaussian-like signal tracts [20] with waveform features and parameters (amplitude, position, duration) that can be directly set by the user.

\section{Contractions}

UC are a crucial part of labour onset and advancement. Their features are important to interpret the progress of labour and the significance of certain FHR abnormalities, such as decelerations. Some authors have developed mathematical models for simulation of UC waveforms but without simultaneous simulation of FHR signals [15].

The basal UC signals were simulated by low-pass filtering a white noise. As for the FHR, a sinusoidal basal tone can be superimposed.

The software here proposed is able also to simulate the contraction events with known features (amplitude, frequency, position, duration). The algorithm for the simulation of these events is similar to that of the accelerations, using Gaussian-like signal tracts with features chosen at user discretion [15].

\section{Floating Line}

The set of events (baseline, accelerations and/or decelerations) added to the FHR signal represents its floating line. To avoid discontinuities in correspondence of the transition from one event to another, they were connected using a weighted mean of their tails.
Finally, for obtaining more realistic signals, we applied a low-pass filter.

\section{Signal loss, Arrhythmias and Outliers}

Some problems in predicting foetal wellbeing through CTG interpretation are related to the visual inspection of clinicians, others to the acquisition system that, in some conditions, can cause the degradation or the loss of the signal. These anomalies, cardiac arrhythmia and artifacts in FHR signals do not represent physiological variations, so that they can be defined outliers [17]. Our software allows the simulation of these outliers. In particular, typical arrhythmias, such as Premature Ventricular Depolarization (PVC), simulated by the succession of two FHR samples, respectively with very high and very low value; losses of signal, for example due to relative movements between the Doppler probe and the foetus; or simply isolated outliers, resembling CTG spikes due to erroneous recording of some FHR sample can be simulated. The signal loss can be simulated as abrupt, groups of samples with values very different from FHR mean, or gradual, in this case the change in FHR value is gradual, i.e. there are sample with intermediate value.

\section{Default Parameters}

In order to verify performances of the software developed, we generated different synthetic CTG signals varying all parameters. However, for some parameters we choose values then considered by default. About power spectrum characteristics, considering that, in comparison with HRV of adult subjects, in FHRV different relationships between LF and HF bands are present, in order to resemble real foetal cases, the following parameters were adopted. LF and $\mathrm{HF}$ were considered to lie between 0.05 and $0.2 \mathrm{~Hz}$, and $0.2-1 \mathrm{~Hz}$, respectively. SVB was fixed to 5 and standard deviation of LF and HF bands to 0.01 and 0.03 , respectively.

FHR mean was set at $140 \mathrm{bpm}$ and SD was heuristically set at 2 (corresponding to a peak-peak amplitude of FHR signal approximately in the range 6-10 bpm).

Besides, a sine baseline with frequency at $0.002 \mathrm{~Hz}$ was added. FHR signals simulated with these default characteristics had duration of about 30 minutes.

The basal UC signals, by default were simulated by low-pass filtering a white noise with mean and SD heuristically set at 10 and 1 respectively.

\section{Visualization}

The software provides two different graphical representations of the simulated signals, the default one employed by Matlab, and one that reproduces the clinical standard. In the latter case, the CTG trace is shown according to the following requirements: CTG paper running speed equal to 1 centimetre $(\mathrm{cm})$ per minute, $25 \mathrm{~cm}$ of recording for each page, FHR range equal to $50-210 \mathrm{bmp}$ and $\mathrm{UC}$ range equal to $0-100$ a.u.

\section{Results}

In this section, we show some examples of simulated FHR and UC signals with different characteristics. Where not specifically indicated, default parameters were used.

In case of clinical visualisation only the first 25 minutes of the signals are shown.

In Figure 1 it is shown according to the default representation a 
Citation: Improta G, Romano M, Ponsiglione A, Bifulco P, Faiella G, et al. (2014) Computerized Cardiotocography: A Software to Generate Synthetic Signals. J Health Med Informat 5: 162. doi:10.4172/2157-7420.1000162

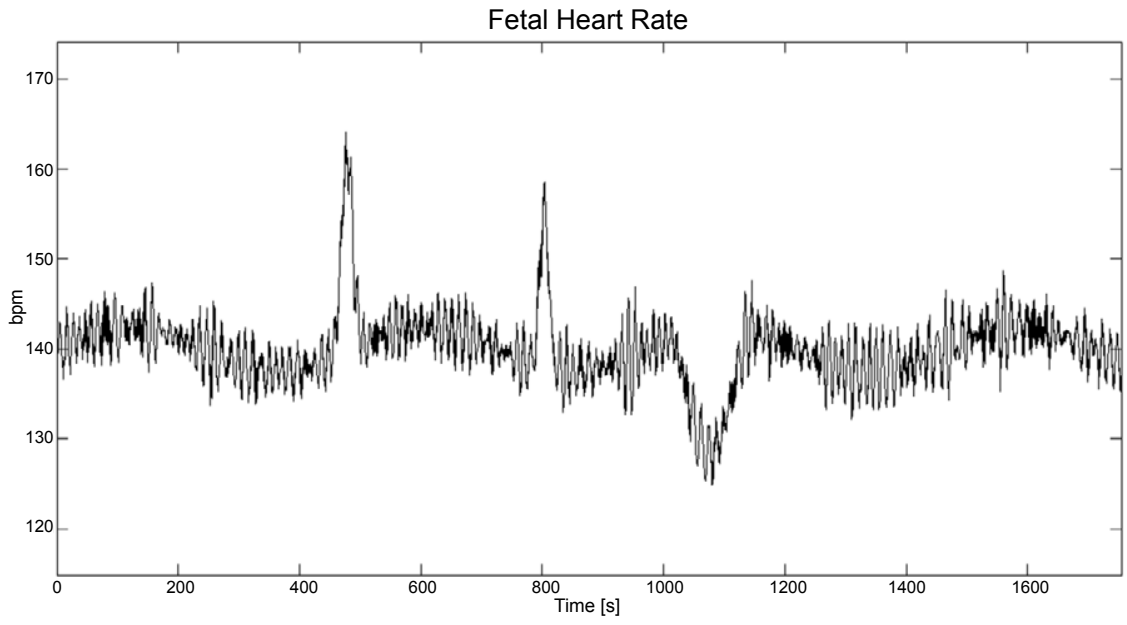

Figure 1: Synthetic FHR, simulated with 2 accelerations and one deceleration.

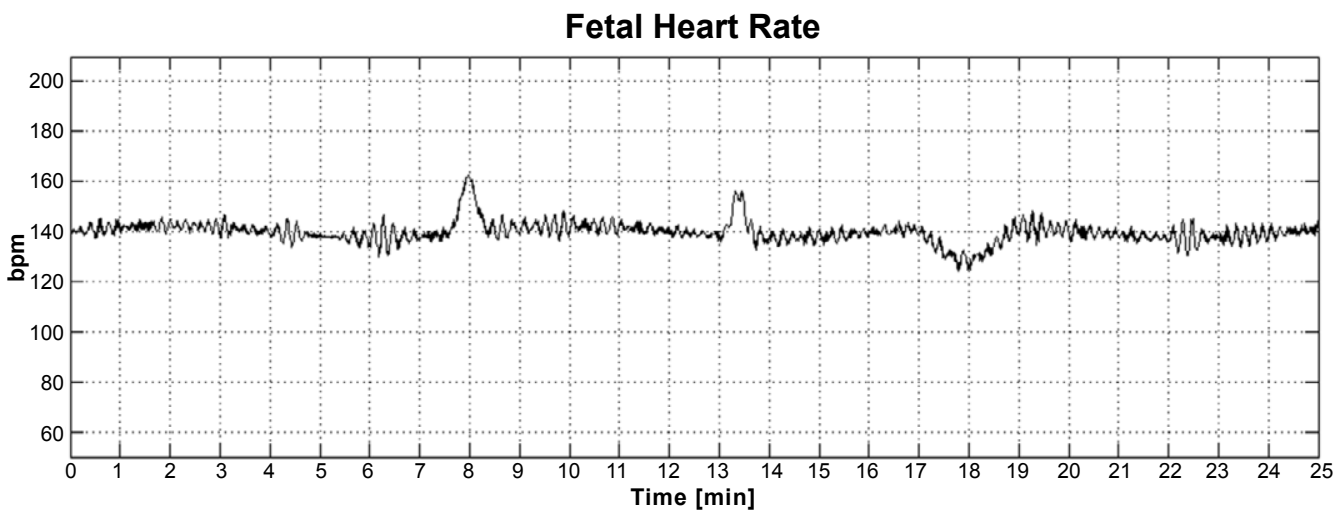

Figure 2: FHR signal of Figure 1 shown according to clinical standard.

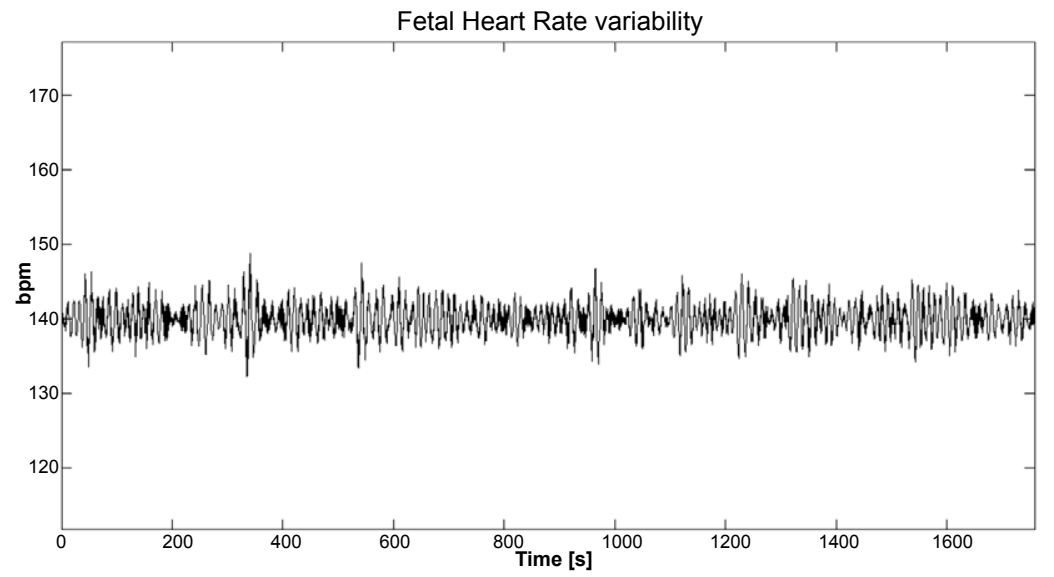

Figure 3: Synthetic signal simulated with a constant baseline and without floatingline superimposed.

synthetic FHR signal simulated by assigning to the various parameters the default values.

An analogous FHR signal shown according to the clinical standard is displayed in Figure 2.
The Figures 3-5 refer to different steps of FHR generation. In particular, a FHR signal simulated with mean equal to $140 \mathrm{bpm}$ and SD equal to 2 is represented in Figure 3. In Figure 4 it is possible to see a floatingline obtained by simulating a sinusoidal baseline slowly varying (default parameters), 2 decelerations and 3 accelerations. The first 
Citation: Improta G, Romano M, Ponsiglione A, Bifulco P, Faiella G, et al. (2014) Computerized Cardiotocography: A Software to Generate Synthetic Signals. J Health Med Informat 5: 162. doi:10.4172/2157-7420.1000162

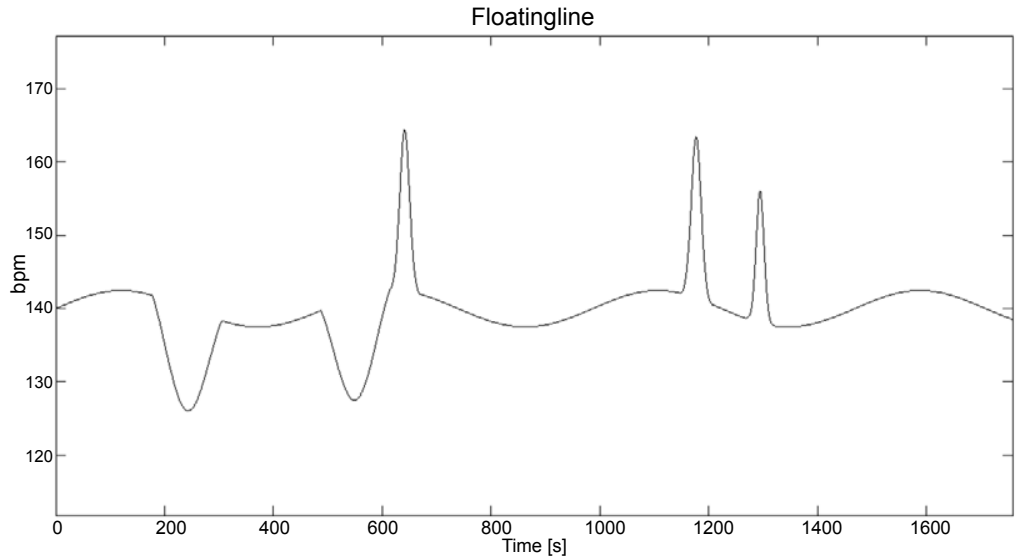

Figure 4: Floatingline simulated and superimposed to the signal of Figure 3 to obtain the FHR signal shown in figure 5.

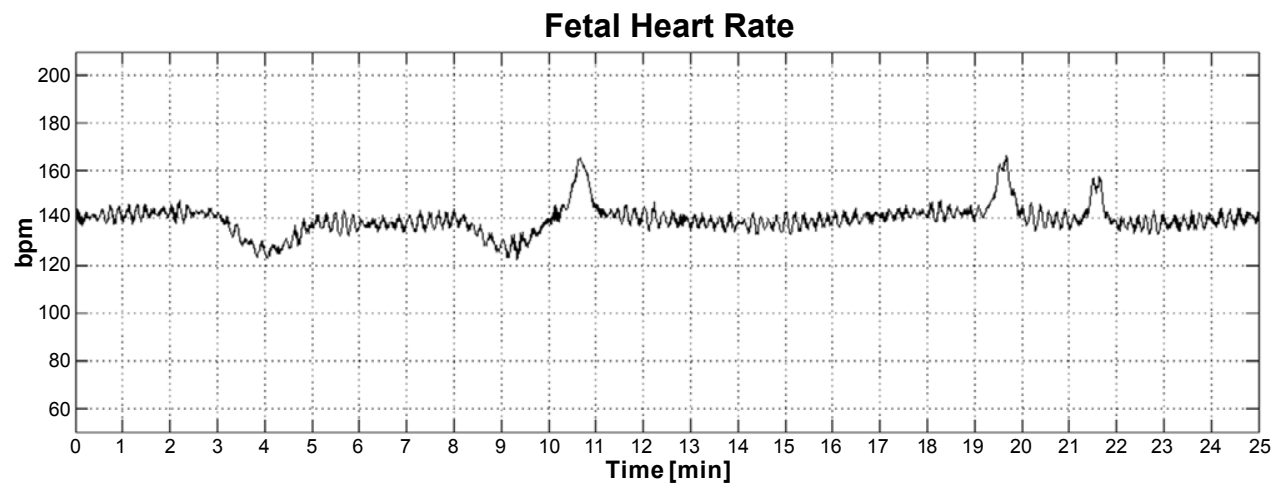

Figure 5: Synthetic FHR, simulated with 2 accelerations, 1 deceleration and a deceleration followed by a recovery phase, shown according to clinical standard. This signal was obtained adding the floatingline shown in Figure 4 to the synthetic signal shown in Figure 3.

Fetal Heart Rate, synthetic signal
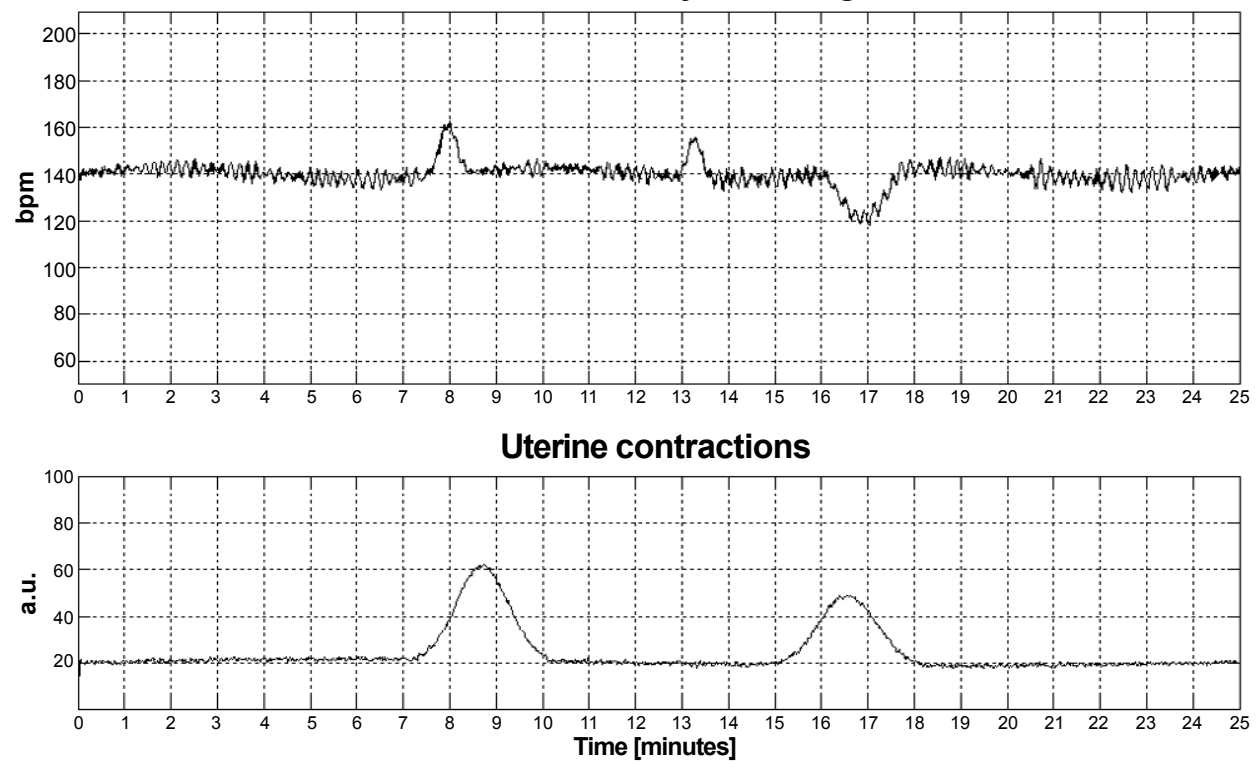

Figure 6: Complete CTGshown according to clinical standard. FHR on the top and UC on the bottom. 
Fetal Heart Rate

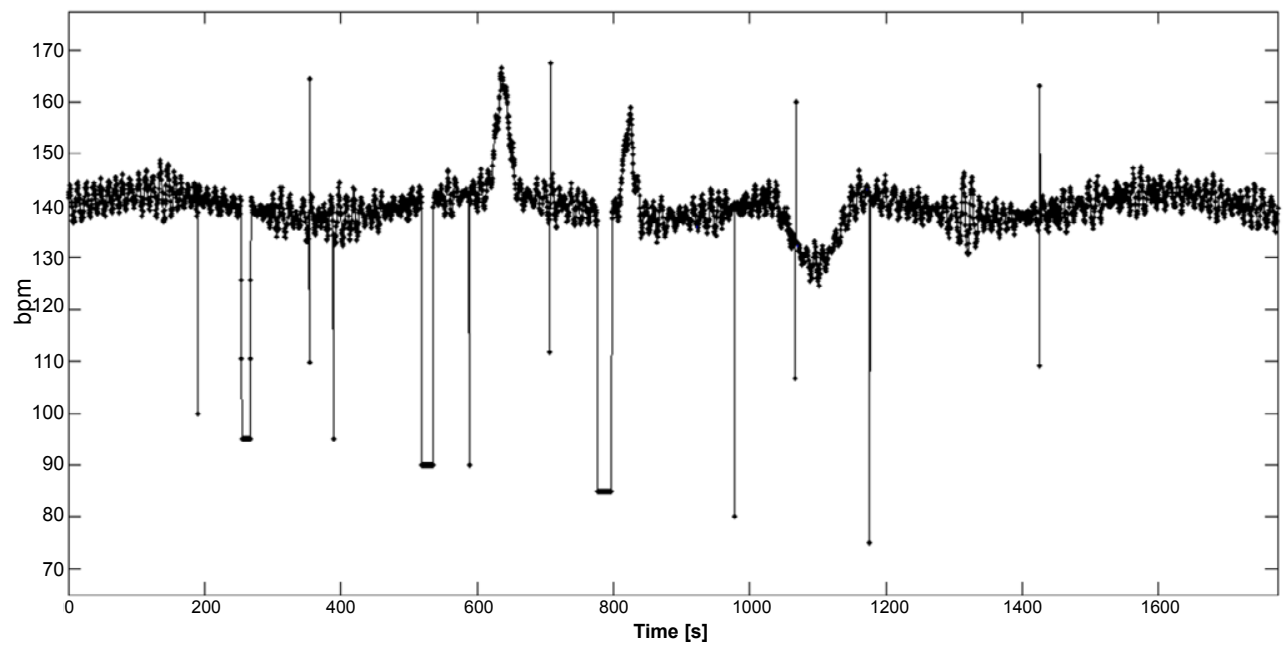

Figure 7: FHR simulated with signal loss (gradual the first, between 250 and $270 \mathrm{~s}$, and abrupt the others, around at 520 and $780 \mathrm{~s})$, arrhythmia (PVC) and isolated outliers.

acceleration was posed just after a deceleration, in order to simulate a foetal recovery phase. In Figure 5 the final FHR signal is shown, it was obtained by the sum of the initial FHR and the floatingline.

In Figure 6 it is shown a complete CTG signal, whit FHR signal on the top, simulated with 2 accelerations and 1 deceleration, and UC signal on the bottom, simulated with 2 uterine contractions. In the FHR signal, the deceleration was generated in correspondence of the second uterine contraction. Both signals were generated using default parameters.

Finally in Figure 7 it is displayed a synthetic FHR signal which shows arrhythmia and outliers. In particular, 3 tracts of signal loss, 4 PVC and 5 isolated outliers were simulated.

\section{Conclusion}

The availability of realistic synthetic CTG signals, with the possibility of easily changing their parameters and features, can be very useful to estimate performances of software employed in computerized cardiotocography. However, at the best of our knowledge, the literature in this field is very poor, except for some isolated cases focused on uterine activity [15].

In this paper, we proposed a software for simulation of FHR and UC signals. The performance of this CTG simulation software are satisfactory, since the synthetic signals generated are comparable to the real ones, meaning that the main features of CTG signals, such as FHR amplitude variability, slow alterations, signal losses etc., can be successfully simulated. Besides, real and synthetic FHR signals present the same characteristics of the main frequency lobes.

Thanks to its versatility, the proposed software can be used in a wide range of applications. The generation of signals representing a typical CTG trace facilitates the comparison of different signal processing methods in order to establish their performances. Moreover, it can be employed to simulate specific events occurring in FHR traces, such as accelerations and decelerations, in order to assess the reliability of algorithms in identifying and classifying such events. Finally, our software can be useful to verify the efficacy of some proposed indexes to estimate particular characteristics of FHR signals, for example variability or sympatho-vagal balance. As further application, it could be employed as a teaching tool for demonstration to medical students and others.

A large set of synthetic FHR series with known features has been already successfully used by the authors to test an algorithm for detection and correction of FHR signals outliers $[18,19]$, to evaluate the performance of an algorithm for the recovery of FHR series out of the zero-order interpolated CTG data [20], to compare several short term variability (STV) indexes performances [21] and to quantitatively evaluate distortion of FHRV spectrum due to different CTG storage rates [22].

\section{Acknowledgement}

This study was partially supported by DRIVE IN2 project - funded by the Italian National Program Piano Operativo Nazionale Ricerca e Competitività 2007/13 and by the project "Sviluppo di sistemi per il monitoraggio fetale domiciliare" funded by "Legge 5" of Campania Region.

The authors would like to thank Dr. Mario Russo of the Gesan S.r.I. for the valuable support provided to the study.

\section{References}

1. Spyridou KK, Hadjileontiadis LJ (2007) Analysis of fetal heart rate in healthy and pathological pregnancies using wavelet-based features. In Engineering in Medicine and Biology Society, 2007. EMBS 2007. 29th Annual International Conference of the IEEE: 1908-1911.

2. Trimbos JB, Keirse MJ (1978) Observer variability in assessment of antepartum cardiotocograms. BrJ Obstet Gynaecol 85: 900-906.

3. Mantel R, Van Geijn HP, Ververs IA, Colenbrander GJ, Kostense PJ (1997) Automated analysis of antepartum fetal heart rate in relation to fetal rest-activity states: a longitudinal study of uncomplicated pregnancies using the Sonicaid System 8000. Eur J of Obstet Gynecol and Reprod Biol 71: 41-51.

4. Maeda K (2014) Fetal Heart Rate Monitoring. J Health Med Informat 5: e112.

5. Maeda K, Arima T, Tatsumura M, Nagasawa T (1980) Computer-aided fetal heart rate analysis and automatic fetal distress diagnosis during labor and pregnancy utilizing external technique in fetal monitoring. Medinfo 80: 12141219

6. Devoe L, Golde S, Kilman Y, Morton D, Shea K et al. (2000) A comparison of visual analyses of intrapartum fetal heart rate tracings according to the new 
Citation: Improta G, Romano M, Ponsiglione A, Bifulco P, Faiella G, et al. (2014) Computerized Cardiotocography: A Software to Generate Synthetic Signals. J Health Med Informat 5: 162. doi:10.4172/2157-7420.1000162

National Institute of Child Health and Human Development guidelines with computer analyses by an automated fetal heart rate monitoring system. Am J Obstet Gynecol 183: 361-366.

7. Bracero LA, Roshanfekr D, Byrne DW (2000). Analysis of antepartum fetal heart rate tracing by physician and computer. J Matern-Fetal Med 9: 181-185.

8. Improta G, Romano M, Amato F, Sansone M, Cesarelli M (2012) Development of a software for automatic analysis of CTG recordings. GNB2012, June 26th29th.

9. Signorini MG, Fanelli A, Magenes G (2014) Monitoring Fetal Heart Rate during Pregnancy: Contributions from Advanced Signal Processing and Wearable Technology. Computational and Mathematical Methods in Medicine.

10. Ayres-de-Campos D, Costa-Santos C, Bernardes J (2005) Prediction of neonatal state by computer analysis of fetal heart rate tracings: the antepartum arm of the SisPorto multicentre validation study. Eur Journal Obstet Gynecol Reprod Biol 118: 52-60.

11. Jafarnia-Dabanloo N, McLernon, DC, Zhang $H$, Ayatollahi A, Johari-Majd $V$ (2007) A modified Zeeman model for producing HRV signals and its application to ECG signal generation. J Theor Biol 244: 180-189.

12. Laguna P, Moody GB, Mark RG (1998) Power spectral density of unevenly sampled data by least-square analysis: performance and application to heart rate signals. IEEE Trans Biomed Eng 45: 698-715.

13. Keselbrener L, Akselrod S (1996) Selective discrete Fourier transform algorithm for time-frequency analysis: method and application on simulated and cardiovascular signals.IEEE Trans Biomed Eng 43: 789-802.

14. Guimaraes HN, Santos RAS (1998) A comparative analysis of preprocessing techniques of cardiac event series for the study of heart rhythm variability using simulated signals. Braz J med Biol Res 31: 421-430.
15. Bastos LF, Van Meurs W, Ayres-de-Campos D (2012) A model for educational simulation of the evolution of uterine contractions during labor. Comput methods programs Biomed. 107: 242-247.

16. Clifford GD, Tarassenko $L$ (2005) Quantifying errors in spectral estimates of HRV due to beat replacement and resampling. IEEE Trans Biomed Eng 52: 630-638.

17. Romano M, Cesarelli M, Bifulco P, Ruffo M, Fratini A et al. (2009) Timefrequency analysis of CTG signals. Current Development in Theory and Applications of Wavelets 3: 169-192.

18. Cesarelli M, Romano M, Bifulco P, Fratini A (2007) Cardiac arrhythmias and artifacts in fetal heart rate signals: detection and correction. In 11th Mediterranean Conference on Medical and Biomedical Engineering and Computing16: 789-792.

19. Romano M, Faiella G, Bifulco P, D'Addio G, Clemente F, et al. (2014) Outliers Detection and Processing in CTG Monitoring. In XIII Mediterranean Conference on Medical and Biological Engineering and Computing 2013: 651-654. Springer International Publishing.

20. Cesarelli M, Romano M, Bifulco P, Fedele F, Bracale M. (2007) An algorithm for the recovery of fetal heart rate series from CTG data. Comput Biol Med 37: 663-669.

21. Cesarelli $M$, Romano $M$, Bifulco $P$ (2009) Comparison of short term variability indexes in cardiotocographic foetal monitoring. Comput Biol Med 39: 106-118.

22. McSharry PE, Clifford GD, Tarassenko L, Smith LA (2003) A dynamical model for generating synthetic electrocardiogram signals. IEEE Trans Biomed Eng 50: 289-294.

23. Toth PP, Jothivijayarani DA (1999) Obstetrics: intrapartum monitoring and management ( $3^{\text {rd }}$ edn). University of lowa Family Practice Handbook. 